Check for updates

Cite this: RSC Adv., 2019, 9, 9145

Received 20th December 2018 Accepted 13th March 2019

DOI: $10.1039 / c 8 r a 10429 h$

rsc.li/rsc-advances

\section{Efficient removal of sulfamerazine (SMR) by ozonation in acetic acid solution after enrichment SMR from water using granular activated carbon}

\begin{abstract}
Youru Yao, (D) ad $\mathrm{Na} \mathrm{Mi}^{a}{ }^{a}$ Yongqing Zhu, ${ }^{a} \mathrm{Li}$ Yin, ${ }^{\mathrm{a}}$ Yong Zhang ${ }^{\mathrm{b}}$ and Shiyin $\mathrm{Li}^{\star \mathrm{ac}}$
Sulfamerazine (SMR) as a persistent organic pollutant in waste streams is of growing environmental concern. This study explores the extraction SMR from water into an acetic acid (AA) solution using granular activated carbon (GAC), and removal of SMR by ozonation in AA solution. Systematic experiments have shown that GAC can be used as an adsorbent to transfer sulfamerazine from water to AA solution. SMR removal efficiency is $99.5 \%$ in $10 \%$ AA aqueous solution, which is better than in water. The removal rate of SMR in the AA solution decreased as the initial molar ratio of SMR and $\mathrm{O}_{3}$ increased. The removal rate of SMR decreased with $\mathrm{Fe}^{3+}$ present in the reactive system. The removal of SMR is dominated by indirect ozonation in water, while the SMR removal is an effect of both direct and indirect ozonation in AA solution. It is a very efficient process for the degradation of SMR in micro polluted water when using combined GAC adsorption-desorption in AA solution and ozonation of the resulting solution.
\end{abstract}

\section{Introduction}

During the last decade, antibiotics as emerging contaminants are of global concern because antibiotic residues in the environment have the ability to expose the public to serious health hazards. ${ }^{1}$ About 100 000-200 000 tons of antibiotics are produced annually for use in animal and human health, agriculture and aquaculture activities, which may aid the antibiotic resistance of microorganisms and destroy the environmental micro-ecosystem. ${ }^{2-4}$ Sulfonamides are one of the most popular groups of antibiotics that have been used for several decades in both human and veterinary medicine, for therapeutic treatment of infections related diseases. ${ }^{5-7}$ However, the residual sulfonamides can enter the environment through various pathways and persist for a long time. Even at low concentrations, the prolonged exposure to the sulfonamide antibiotics may cause harmful effects on humans and aquatic life, such as green algae, Daphnia magna, and lemna minor.,

Considering serious menace to the environment caused by low-concentration antibiotics in the aquatic system, the development of efficient removal methods in advanced water treatment is becoming an urgent matter. ${ }^{10}$ The removal of antibiotics has been tested using various processes such the photo-Fenton process, photochemical oxidation technique, UV-

${ }^{a}$ School of Environment, Nanjing Normal University, Nanjing 210023, China. E-mail: lishiyin@njnu.edu.cn

${ }^{b}$ Department of Geological Sciences, University of Alabama, Tuscaloosa, AL 35487, USA cJiangsu Center for Collaborative Innovation in Geographical Information Resource Development and Application, Nanjing, 210023, China

${ }^{d}$ School of Geography and Tourism, Anhui Normal University, Wuhu, 241003, China irradiation, electrochemical processes and adsorption. ${ }^{11-18}$ However, electrochemical and photo-Fenton processes seem suitable to treat toxic wastewaters with high concentrations of antibiotics; other techniques, to a certain extent, are blamed for the high investment and processing cost and low qualified rate in wastewater treatment., ${ }^{2,19}$ Adsorption has become an emerging method in waste removal because of its simplicity, low operation cost..$^{20}$ The most widely reported adsorbents for antibiotic include ACs, CNTs, bentonite, ion exchange resins, BCs and so on. Considering the easy availability of raw materials, high surface area, degree of micro porosity and high adsorption capacity, activated carbon was used to adsorb sulfamerazine (SMR) in wastewater containing organic contaminants, ${ }^{21,22}$ and desorbed SMR from activated carbon by acid solution. Nevertheless, it is not appropriate to recycle or reuse acidic solutions containing antibiotics and other mixtures to desorb antibiotics from activated carbon. This method is not appropriate in complex water, containing low-concentration antibiotics, due to low recovery value.

Ozonation is a method for removing antibiotics and recalcitrant pollutants from wastewater, which has unique advantages like easy equipment, easy operation and high speed. ${ }^{23}$ It is widely acknowledged that ozone can react with various organic compounds in aqueous solution in two ways: a direct reaction with molecular ozone or a radical reaction involving hydroxyl radicals induced by ozone decomposition. Therefore, the removal of antibiotics in water by ozonation is affected by many factors, including solution $\mathrm{pH}$, inorganic anions and cations, dissolved organic matter, $\cdot \mathrm{OH}$ scavenger and the type of water matrices. ${ }^{24,25}$ Conventional treatment systems dissolve ozone directly into a wastewater by gas/liquid contact. However, 
ozonation in water is not a universally efficient process, because current ozone-generating technology is incapable of producing gas concentrations of more than several percent and the solubility of ozone in water is relatively poor (approximately $0.2 \mathrm{mg} \mathrm{L}^{-1}$ per $\mathrm{mg} \mathrm{L}^{-1}$ in contacting gas phase). ${ }^{26}$ Due to poor oxidation selectivity and, particularly, a low utilization ratio and the high energy consumption of ozone in conventional water/ gas ozonation systems, more recent techniques increase production costs to added catalysts and/or irradiation to the oxidation medium to improve oxidation efficiency. ${ }^{27,28} \mathrm{G} . \mathrm{Li}$ et $a l .{ }^{22}$ studies have shown that the removal efficiency was more than $80 \%$ for the studied SMR by an integrated ozonation and biological activated carbon filtration $\left(\mathrm{O}_{3}\right.$-BAC) process, under the concentration of SMR is $1000 \mathrm{ng} \mathrm{L^{-1 }}$ at ozone doses of 0.5 and $2.5 \mathrm{mg} \mathrm{L}^{-1}$. The removal rate at high concentrations of SMR was lower than that of low concentrations. As a result, these factors combine to impede the broad application of ozonation in wastewater treatment. ${ }^{29}$

In order to solve the above problems, as conventional adsorbent, granular activated carbon (GAC) was used to extract organic pollutants from wastewater. GAC loaded with organic pollutants was washed with organic solvents such as acetic acid (AA), acetone and tert-butyl alcohol. Organic pollutants were decomposed by ozonation more efficiently in organic solvents than those in water. In addition, using organic solvents prevents the unnecessary formation of ozonation by-products that occur in water. Because organic solvents or saturated carboxylic acids reacts slowly with ozone and in many cases not be completely oxidized, ${ }^{15}$ they can be reused to extract organic pollutants from adsorbents loaded with organic pollutants. In the previous studies, ozonation of chlorinated organic compounds in organic solvents as an effective process, was used in the efficient treatment of wastewater..$^{30-32}$

There are little comprehensive studies known about the removal of SMR with ozonation in AA solution after extracted SMR using GAC from wastewater. In this paper, the purpose of the study is to investigate the primary factors that may influence the effect of ozonation removal of SMR in AA solution, with the aim of providing a critical application for the practical treatment of wastewater containing low-concentration antibiotics.

\section{Materials and methods}

\subsection{Materials}

The reagents used in this study include methanol (HPLC, TEDIA Company), sulfamerazine (SMR, Nanjing Boquan Technology Co., Ltd., purity higher than 95\%) and acetic acid (AR, Sinopharm Chemical Reagent CO., Ltd). The GAC was purchased from Tianjing Activated Carbon Co., Ltd (Tianjing, China). The GAC was pulverized into a 100 mesh powder, and then was soaked in $0.01 \mathrm{~mol} \mathrm{~L}^{-1} \mathrm{HCl}$ solution for 72 hours. The samples were then rinsed with deionized water to a neutral $\mathrm{pH}$, dried at $120{ }^{\circ} \mathrm{C}$ in an oven and stored in a desiccator. Ozone generator (3-10 $\mathrm{g} \mathrm{h}^{-1}$, Nanjing Woer Technology Co., Ltd.) with industrial oxygen $(99.5 \%)$ was used to provide the source gas. The concentration of SMR was measured using a liquid chromatographer (LC-100P, Shanghai Wufeng Scientific Instruments Co., Ltd.).

\subsection{Adsorption and desorption experiments}

Adsorption experiment. The adsorption kinetics study of GAC was performed by adding $0.01,0.02,0.03,0.04,0.05,0.06 \mathrm{~g}$ samples and $100 \mathrm{~mL} 150 \mathrm{mg} \mathrm{L}^{-1} \mathrm{SMR}$ solutions to $250 \mathrm{~mL}$ glass vials. $\mathrm{HCl}$ was added drop wise to the solution for adjusting the $\mathrm{pH}$ to 2.1. Then, all samples were shaken at a constant agitation speed $(220 \mathrm{rpm})$ at $25 \pm 0.5^{\circ} \mathrm{C}$. Samples were obtained at 5, 10, $20,40,60,90,120,180,360,540,720,1080,1440,2880$, $7200 \mathrm{~min}$, respectively. ${ }^{33}$ After reaching equilibrium, the solution was separated from the sorbent by centrifugation at $13000 \mathrm{rpm}$ for $5 \mathrm{~min}$ and filtered with a $0.45 \mu \mathrm{m}$ pore size syringe filter. Freundlich isotherms were taken to analyze the adsorption capacity of GAC for SMR.

Extraction experiments. Acetic acid was used to desorb SMR from GAC. GAC $\left(0.4 \mathrm{~g} \mathrm{~L}^{-1}\right)$ containing SMR was placed in a $50 \mathrm{~mL}$ vial with $40 \mathrm{~mL}$ of AA solution $(5 \%, 10 \%$ and $30 \%(\mathrm{v} / \mathrm{v}))$. The vial was sealed with a Teflon coated butyl rubber septum and shaken at $120 \mathrm{rpm}$ for 24 hours at $25{ }^{\circ} \mathrm{C}$. Extract solution was separated by centrifugation. After three extractions, three rounds of extract solution containing SMR were combined for ozonation. After cleaning with water, GAC was used for next adsorption. After ozonation, acetic acid solution was used for the next extraction of SMR. GAC was not involved in the oxidative degradation of SMR by ozone in AA solution. After cleaning with water, GAC was used for next adsorption.

In this work, pure oxygen was used as source to generate ozone constantly. Acetic acid solution $(150 \mathrm{~mL})$ containing SMR (200 $\mathrm{mg} \mathrm{L}^{-1}$ ) was placed in the reactor with a G3 glass gas diffuser, where the available capacity of the reactor was $1 \mathrm{~L}$. Thus, $\mathrm{O}_{3}$ was bubbled from the gas diffuser into the glass reactor at a speed of $0.05 \mathrm{~m}^{3} \mathrm{~h}^{-1}, 4.81 \mathrm{mg} \mathrm{O}$ per min. Vaporliquid contact oxidation between ozone and SMR occurred in the glass reactor. Similar experiments were carried out with AA solutions containing tertiary butyl alcohol (TBA) $20 \mathrm{mmol} \mathrm{L}^{-1}$ and $\mathrm{Fe}^{3+}$ respectively. Blank solution, without AA, was prepared by using sulfuric acid to adjust the $\mathrm{pH}$ to 2.0. The whole experiment was conducted in a closed environment without lights. Samples were collected at a certain time interval during each reaction, and a $5 \mu \mathrm{L}, 0.025 \mathrm{~mol} \mathrm{~L}^{-1} \mathrm{Na}_{2} \mathrm{~S}_{2} \mathrm{O}_{3}$ solution was added to each sample immediately after collection, to terminate the oxidation reaction.

\subsection{Analytical methods}

The textural properties of the GAC were characterized using scanning electron microscope (SEM) (JSM-5610LV, Jeol Ltd., Japan). The concentration of ozone in solution was determined by the indigo method. The SMR in solution was determined by liquid chromatography with a UV/Vis detector and an ODS-C 18 column. The detection was made with a $1.0 \mathrm{~mL} \mathrm{~min}^{-1}$ mobile phase (with a concentration of $0.01 \mathrm{~mol} \mathrm{~L}^{-1}$, a $\mathrm{pH}$ of 3.0 , the ratio between phosphate buffer solution and acetonitrile of 20/ 80 , and $\mathrm{H}_{3} \mathrm{PO}_{4}$ used to adjust the $\mathrm{pH}$ of the water phase) at $280 \mathrm{~nm}$, and a column temperature of $30{ }^{\circ} \mathrm{C}$ for SMR. 
Calibration curves remained linear through the detection limit range from $0.02 \mathrm{mg} \mathrm{L}^{-1}$ to at least $5 \mathrm{mg} \mathrm{L}^{-1}$. The average rate of SMR recovery was $98.5 \%$. All samples were measured in triplicates. Relative standard deviation was less than $10 \%$. The injection volume was $0.02 \mathrm{~mL}$.

HPLC-MS methods have been were observed during the ozonation degradation of sulfamerazine in reversed-phase mode on an Agilent ZORBAX SB-C $\mathrm{C}_{18}$ column $(1.8 \mu \mathrm{m}, 50 \times 4.6$ mm; Agilent) using a 1260 Infinity LC system (Agilent, USA). Mobile phases consisted of $0.1 \%$ trifluoroacetic acid (TFA) in water (eluent A) and acetonitrile (eluent B). The following gradient was applied: $0 \mathrm{~min}-20 \% \mathrm{~B}, 1 \mathrm{~min}-20 \% \mathrm{~B}, 3 \mathrm{~min}-$ $80 \%$ B, 4 min $-80 \%$ B, $4.1 \min -20 \%$ B, $7.1 \min -20 \%$ B. The MS was operated in the positive ESI mode. The MS parameters were as follow: capillary $4500 \mathrm{~V}$, source temperature $100{ }^{\circ} \mathrm{C}$, desolvation temperature $350{ }^{\circ} \mathrm{C}, \mathrm{RF}$ voltage $0.2 \mathrm{~V}$, cone backflush flow $50 \mathrm{~L} \mathrm{~h}^{-1}$, cracking voltage $135 \mathrm{~V}$, sheath gas (nitrogen) $0.75 \mathrm{~L} \mathrm{~min}^{-1}$, auxiliary gas (nitrogen) gas $0.15 \mathrm{~L} \mathrm{~min}^{-1}$. The MS spectra were acquired over an $\mathrm{m} / \mathrm{z}$ range of $50-400$.

\section{Results and discussion}

\subsection{Adsorption and extraction of SMR by GAC}

The porous structure of the surface can be seen by SEM of GAC (Fig. 1a). Moreover, a large number of porous and loose structures can be observed on the surface of the GAC after being soaked with acid (Fig. 1b and c). GAC has gained widespread use for the adsorption of pollutants main due to its large surface area, porous structure and highly active surface. ${ }^{34}$ Therefore, the strong adsorption ability of GAC is utilized for SMR adsorption experiments.

The adsorptive capacity of GAC to SMR at different concentrations was different (Fig. 2a). There was a positive correlation between concentration of GAC and adsorption capacity. When the concentration of GAC is $0.6 \mathrm{~g} \mathrm{~L}^{-1}$, the maximum adsorption capacity can reach $165.67 \mathrm{mg} \mathrm{g}^{-1}$ compared with other concentration conditions. The amount SMR adsorbed by GAC varies with time. But the adsorption will reach equilibrium state after 1440 min of reaction time. Using the Freundlich isotherm to evaluate the adsorption of SMR by GAC. ${ }^{19} \mathrm{Fig} .2 \mathrm{~b}$ presents the Langmuir isotherm of GAC with SMR is shown. The correlation coefficient of fitting is 0.998 . The results show that GAC has a good ability to adsorb SMR from water.

The GAC adsorbed with SMR was extracted in $10 \%(\mathrm{v} / \mathrm{v})$ AA solution for $4 \mathrm{~h}$, then the concentration of SMR dissolved in AA solution was determined. The extraction rate of SMR in $10 \%(\mathrm{v} /$ v) AA solution were $93.92 \%, 92.24 \%, 93.32 \%, 85.42 \%, 87.89 \%$ and $80.56 \%$, respectively, when the concentration of GAC were $0.1,0.2,0.3,0.4,0.5,0.6 \mathrm{~g} \mathrm{~L}^{-1}$ (Fig. 2c).

To better evaluate changes in adsorbability and extractability, the adsorption and extraction of SMR was repeated five times. Fig. $2 \mathrm{~d}$ reveals the amount of SMR adsorbed by and extracted from GAC. GAC adsorption capacity were 125.78, $85.52,83.26,84.69$ and $85.5 \mathrm{mg} \mathrm{g}^{-1}$ and the extraction capacity were $120.36,83.62,80.91,82.37,83.68 \mathrm{mg} \mathrm{g}^{-1}$ in five adsorption-extraction cycles, respectively. The first step has a considerably higher amount of adsorption than the second step (67.99\% of first step). This may be due to the fact that some AA

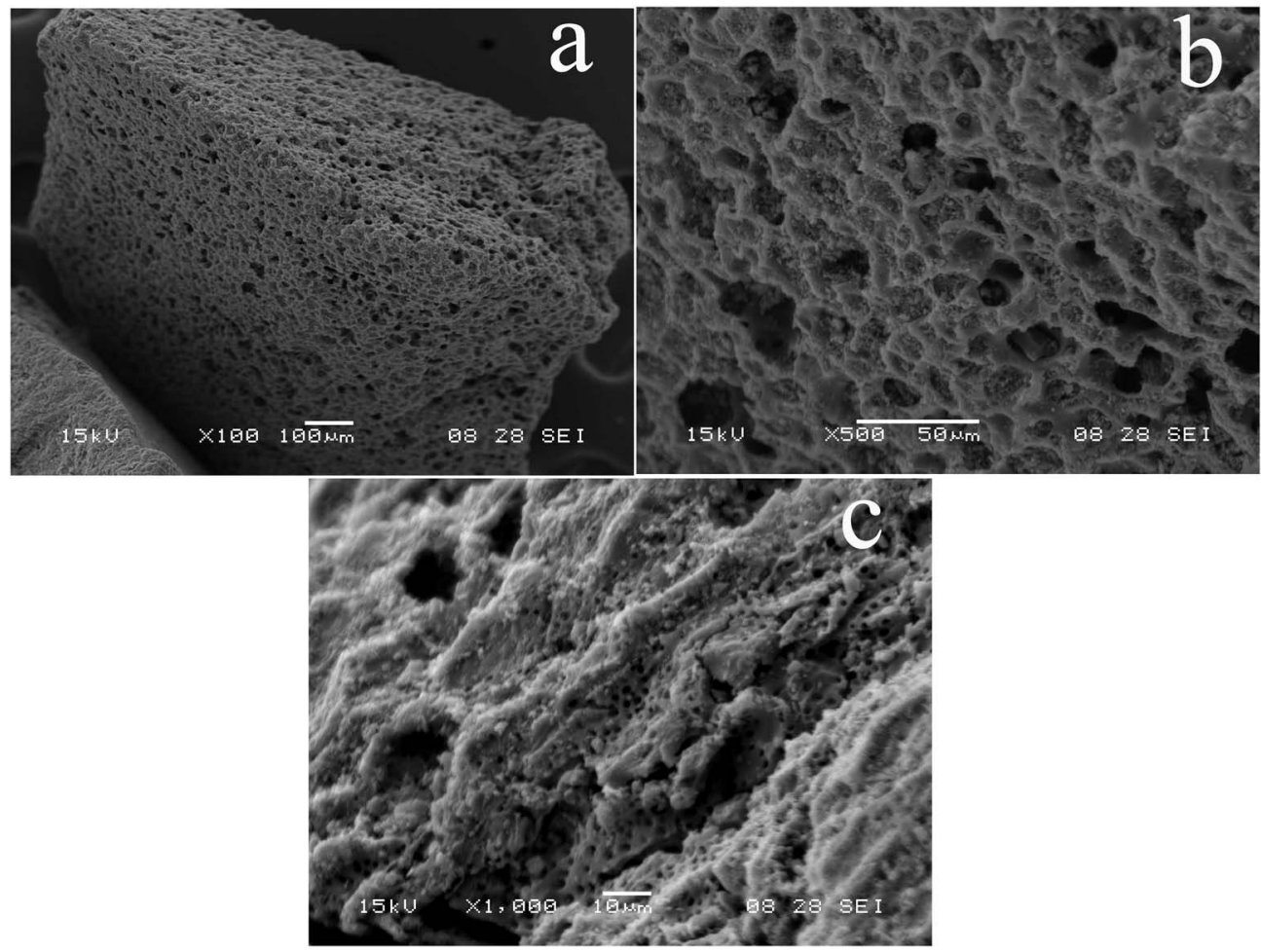

Fig. 1 Scanning electron microscope of granular activated carbon. (a) scale bar is $100 \mu \mathrm{m}$, (b) scale bar is $500 \mu \mathrm{m}$, (c) scale bar is $1000 \mu \mathrm{m}$. 
a

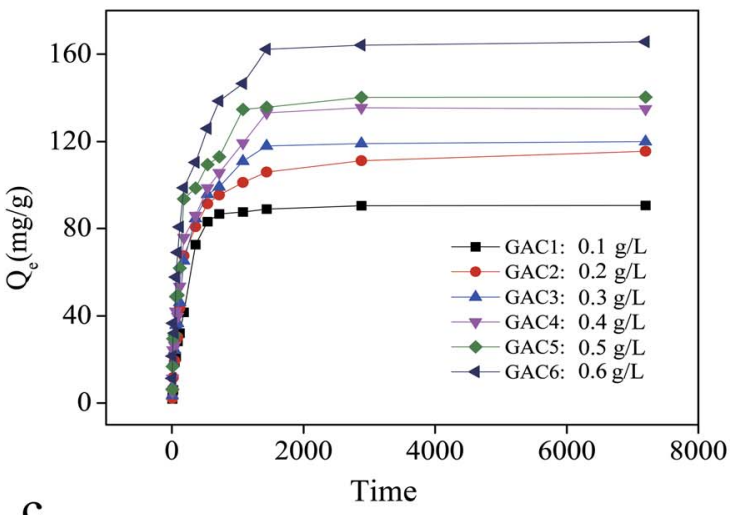

c

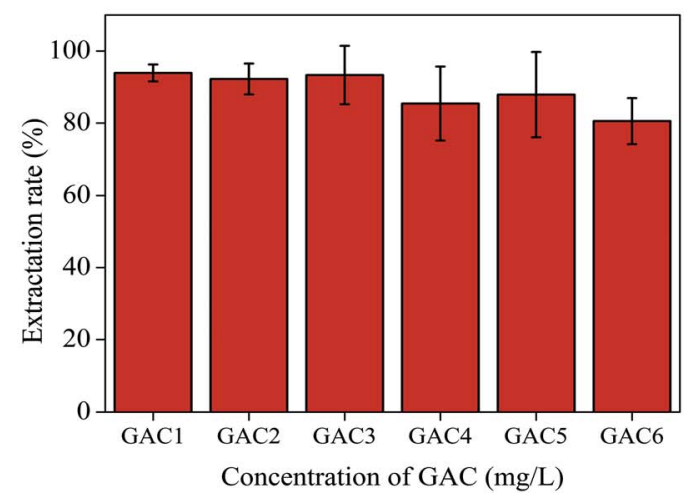

$\mathrm{b}$
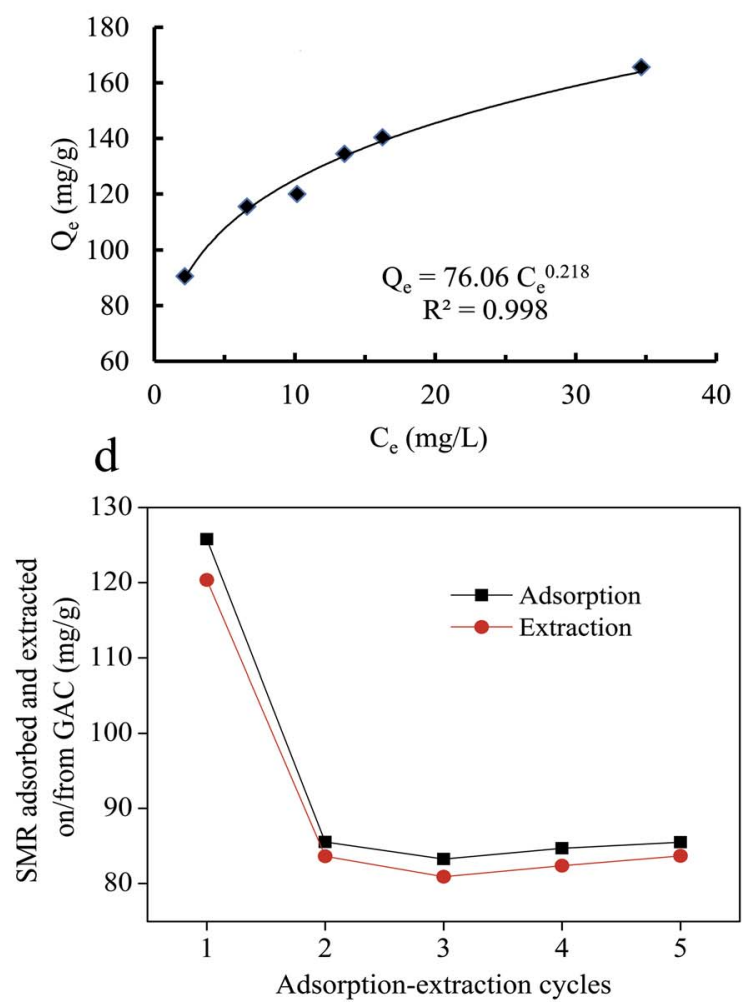

Fig. 2 (a) Effect of time on the adsorption of SMR with different initial GAC concentrations ( $150 \mathrm{mg} \mathrm{L}^{-1}$ of $\mathrm{SMR}, 25 \pm 0.5^{\circ} \mathrm{C}$ ), (b) Freundlich adsorption isotherm of GAC for SMR, (c) the extraction rate of SMR from GAC in $10 \%$ (v/v) AA solution under different concentration of GAC, (d) the relationship between the amount of SMR adsorbed by, and extracted from GAC (10\% (v/v) AA solution).

solution occupies the holes on the surface of GAC after the first step of extraction, which results in the decrease of adsorption sites on the surface of GAC. However, it was observed that the following adsorption and extraction remained relatively stable. Regarding the ratio of extraction and adsorption, it held almost constant for all five steps and the SMR adsorbed on GAC was completely extracted after the second step. The ratio of each extraction to adsorption is $0.96,0.98,0.97,0.97$ and 0.98 , respectively. The results show that the SMR adsorbed by GAC in water can be extracted well in AA solution under the condition of repeated use. These results indicate that GAC can be used as an adsorbent to transfer SMR from water in AA solution.

\subsection{Effects of acetic acid concentration on the removal of SMR}

As can be seen from Fig. 3, SMR removal in AA solution is more efficient than that in water as the initial concentration of SMR is $200 \mathrm{mg} \mathrm{L}^{-1}$, the ozone flow rate is $0.05 \mathrm{~m}^{3} \mathrm{~h}^{-1}$, and the ozone

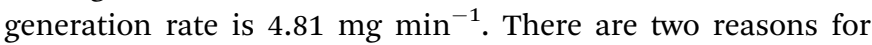
removal rate of SMR is higher in AA solution than in water: AA reduces the ozone decomposition rate, ozone has a larger solubility in AA solution. Furthermore, the SMR removal efficiency is better in $5 \%$ AA solution than that of in $10 \%$ or $30 \%$ AA solution. This phenomenon could be explained that there may exist concurrent direct and indirect ozone oxidation reactions when ozone is in the acidic medium. Thus, the increase in the concentration of acetic acid gradually reduces the ozone oxidation reaction rate, ${ }^{35}$ which further reduces the overall removal rate of SMR. According to account the extraction SMR from GAC, AA concentration was fixed at $10 \%$ in the following

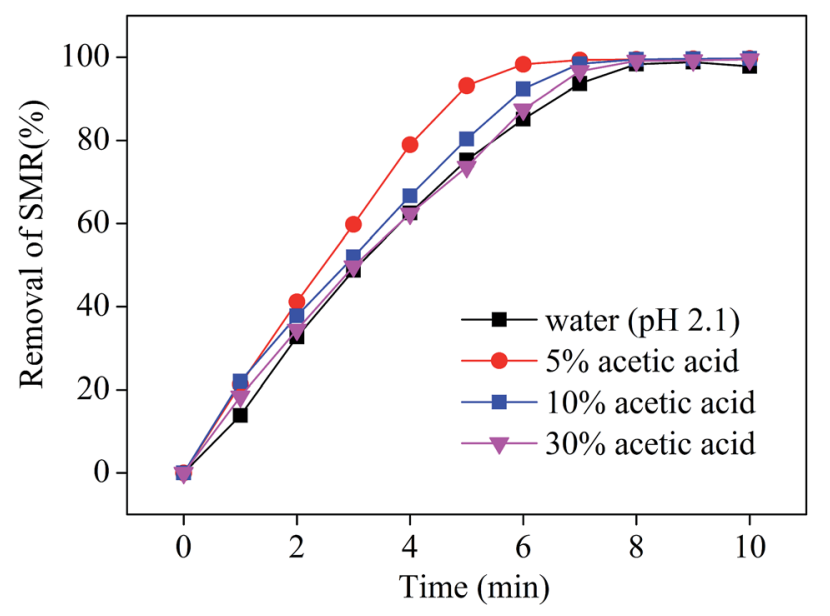

Fig. 3 Effects of concentration of acetic acid on the removal of SMR by ozonation. 
experiment. A very important point is that the removal of SMR does not mean its combination with AA but its degradation. Two intermediates were observed during the ozonation degradation of sulfamerazine, which are completely degraded during the ozonation for reactive time more than 8 minutes. No other intermediates were detected by HPLC-MS when reactive time more than 8 minutes. In addition, it is important to point out that TOC can not be used in this test to monitor mineralization of the SMR because of the huge concentration of AA.

\subsection{Effects of the initial molar ratio of $\mathrm{SMR}$ and $\mathrm{O}_{3}$ on the removal of SMR}

To investigate the effect of the initial molar ratio of SMR and $\mathrm{O}_{3}$ on TC removal, the different $\mathrm{SMR} / \mathrm{O}_{3}$ ratios were given $(1.21: 1$, $1.51: 1,1.82: 1$ and $2.12: 1$, respectively) in the $10 \%$ AA solution with the $\mathrm{O}_{3}$ flux at $0.05 \mathrm{~m}^{3} \mathrm{~h}^{-1}$ and $\mathrm{O}_{3}$ generation rate at $4.81 \mathrm{mg} \mathrm{min}^{-1}$. As shown in Fig. 4 shows that the removal rate of SMR in the AA solution decreases as the initial molar ratio of SMR and $\mathrm{O}_{3}$ increases. More $\mathrm{O}_{3}$ is needed to be consumed as a result of the initial molar ratio of SMR and $\mathrm{O}_{3}$ increases. ${ }^{36} \mathrm{In}$ the meantime the concentration of intermediates of SMR oxidation increases, further consuming $\mathrm{O}_{3} \cdot{ }^{37}$ The results showed that the removal efficiency of SMR was worse with the increase of the ratio of SMR and $\mathrm{O}_{3}$. SMR with high concentration extracted into AA solution can be better removed by increasing ozone concentration. However, considering the economic benefits, SMR can be better removed when the ratio of SMR and $\mathrm{O}_{3}$ is $1.82: 1$ than that of other ratio conditions.

\subsection{Effects of the free radical scavenger on the removal of SMR}

Free radical scavengers are compounds which compete with other hazardous organics for $\cdot \mathrm{OH}$ during single-phase ozonation reactions, such as tertiary butyl alcohol (TBA). It was found that TBA can significantly reduces the degradation efficiency of pollutants in the process of treating industrial wastewater. ${ }^{38}$ In this study, TBA with a concentration of $20 \mathrm{mmol} \mathrm{L}^{-1}$ in both

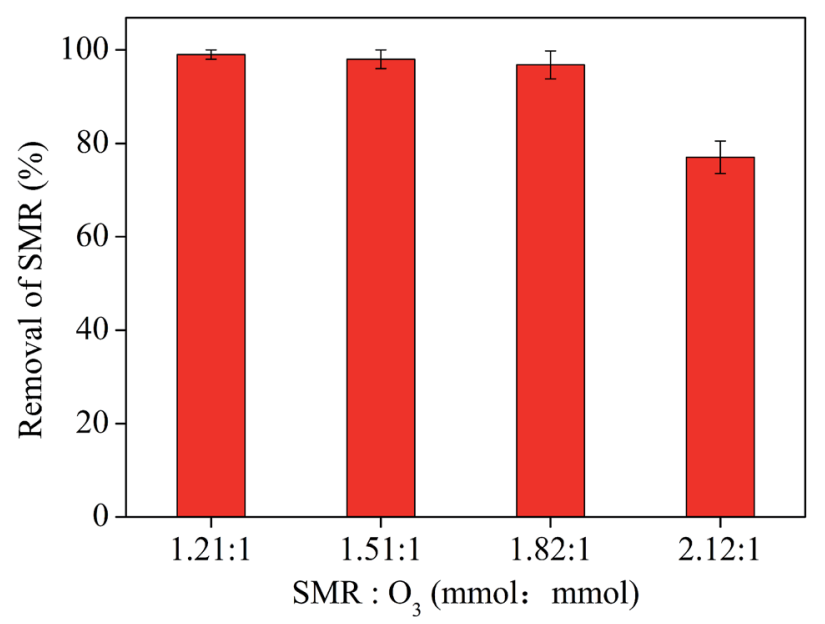

Fig. 4 Effect of the initial molar ratio of $\mathrm{SMR}$ and $\mathrm{O}_{3}$ on the removal of SMR in acetic acid aqueous solution. water (adjusting $\mathrm{pH}$ to 2.1 with sulfuric acid) and 10\% AA solutions was used to investigate the effects of a free radical scavenger on the removal of SMR. This effect was evaluated with an initial SMR concentration of $200 \mathrm{mg} \mathrm{L}^{-1}$, an $\mathrm{O}_{3}$ flux of 0.05 $\mathrm{m}^{3} \mathrm{~h}^{-1}$, and an ozone generation rate of $4.81 \mathrm{mg} \mathrm{min}^{-1}$. The effect of TBA on the removal of SMR in the $10 \%$ AA solution and aqueous phases is depicted in Fig. 5. Compared with the reactive system of no TBA, SMR removal by ozonation is considerably inhibited by the addition of TBA at the beginning of the process. In the presence of TBA, as a whole, the inhibition effect of on SMR removal is similar in both AA solution and water. This results might be due to the strong affinity between TBA and . $\mathrm{OH}\left(k=5 \times 10^{8} \mathrm{~mol} \mathrm{dm}{ }^{-3} \mathrm{~s}^{-1}\right)$, which inhibits the production of $\cdot \mathrm{OH}$ and further inhibits indirect ozonation. ${ }^{39}$ The above result illustrates that SMR removal in water is dominated by indirect ozonation, while the SMR removal in AA solution is an effect of both direct and indirect ozonation. ${ }^{40}$

\subsection{Effects of metal ion on the removal of SMR by ozonation in acetic acid}

In previous work it was found that conventional metal ions with invariable valence had little catalytic effect on ozonation, while obvious changes in ozonation rate were observed in the reactive system containing ions with variable valence. ${ }^{41}$ The kinetics and yield of $\cdot \mathrm{OH}$ formation in ozone decomposition were affected by the addition of variable valence metal ions under different conditions. ${ }^{42}$ Various metals can present in AA solution when AA is used as a solvent for the desorption process. Iron is a transition metal widely existing in natural and waste waters. In this work, a variable valence, iron was selected to investigate the effect of metal ions on SMR removal by ozonation in $10 \% \mathrm{AA}$ solution. $\mathrm{Fe}^{3+}$ was added to the solution with an initial SMR concentration of $200 \mathrm{mg} \mathrm{L}^{-1}, \mathrm{O}_{3}$ flux of $0.05 \mathrm{~m}^{3} \mathrm{~h}^{-1}$, and $\mathrm{O}_{3}$

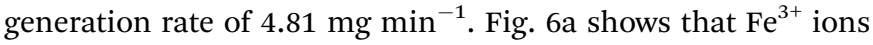
significantly reduces the SMR removal rate by ozonation in AA solution. Some researchers have pointed that the metal-tetracycline complex has low reactivity with ozone because of the

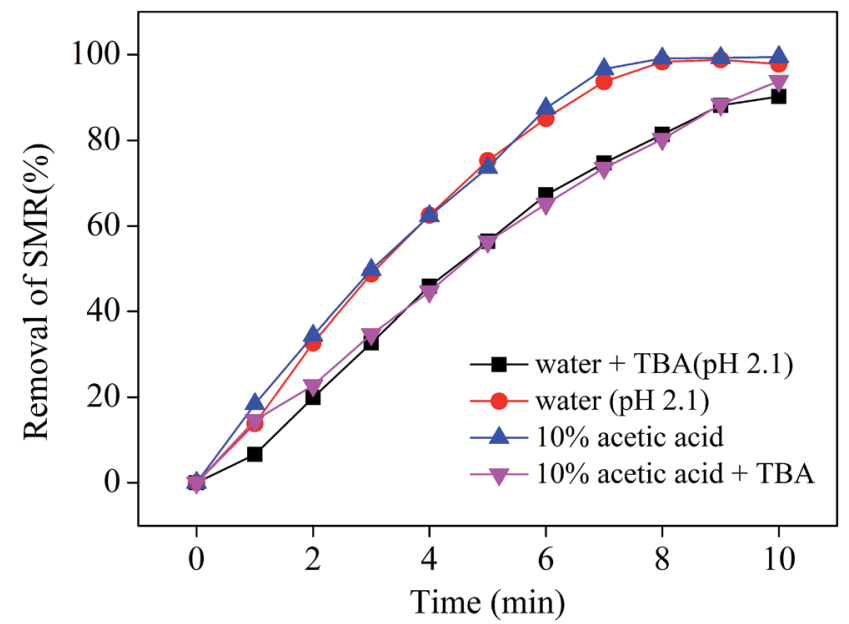

Fig. 5 Effect of free radical scavenger on the removal of SMR by ozonation in 10\% acetic acid and aqueous phases. 

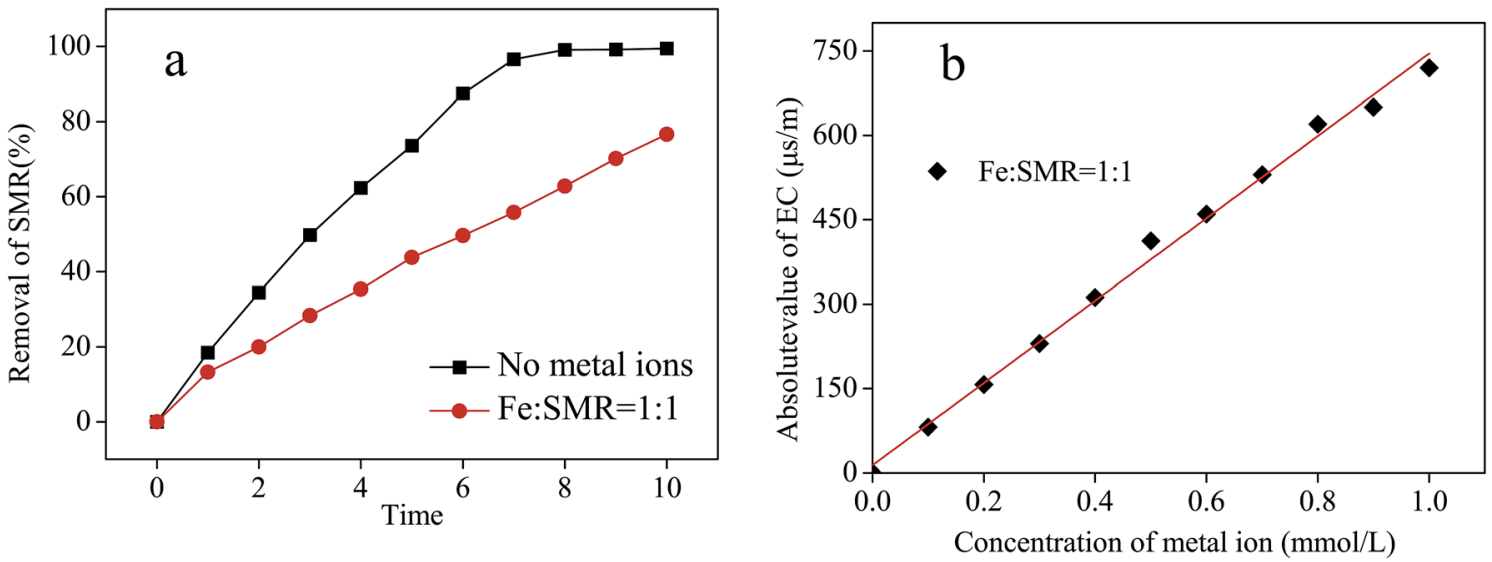

Fig. 6 Effect of metal ion on SMR removal in acetic acid solution (a) and the relationship between the value of electrical conductivity and the concentration of $\mathrm{Fe}^{3+}$ (b).

formation of stable complexes between tetracycline and metal ions. $^{32,43,44}$ In contrast with water, the effect of $\mathrm{Fe}^{3+}$ ions on ozonation in AA solution implies that the ozonation mechanism of SMR in AA solution is not same as that in water. For this reason, experiments were carried out to determine electrical conductivity (EC) of a $10 \%$ AA solution containing SMR and $\mathrm{Fe}^{3+}$, which can investigate the interaction between metal ions and SMR. The difference between the electrical conductivity of acetic acid containing SMR and that of acetic acid without SMR was defined as the absolute value of electrical conductivity. The relationship between the absolute value of EC and the concentration of $\mathrm{Fe}^{3+}$ ions is shown in Fig. 6b. Compared with the system of without SMR, the value of electrical conductivity increased with the increase of $\mathrm{Fe}^{3+}$ ions. This might be connected to the fact that metal-SMR complex was not produced in AA solution with the presence of $\mathrm{Fe}^{3+}$.

The removal efficiency of SMR was inhibited by the increase of iron ion concentration, with a reduction rate of about 22.83 (Fig. 6a). Some studies have shown that the formation rate of . OH by ozone in aqueous solution with iron ions is higher than that in the solution without iron ions, under neutral and alkaline conditions. ${ }^{42}$ However, in this study, SMR was oxidized by ozone in AA solution under acidic conditions. The degradation mechanism of SMR in acetic acid solution may be different from that in water. The mechanism of iron affecting ozonation in AA solution needs further study.

\subsection{Effect of reusing AA solvent on the desorption and removal of SMR}

To investigate the effect of reusing AA solution on the desorption and removal of SMR, the AA solution was reused in desorption and experiments. Effect of reusing AA solution on desorption and removal of SMR is showed in Fig. 7. During the process of desorption and removal of SMR, the rate of desorption and the rate of removal reach $89.1 \%$ and $90.3 \%$ respectively, after AA solution was repeated for five desorption cycles. Therefore, as to the concentration of SMR by using by GAC, AA solution is economically sound, efficient and recyclable for desorption and ozonation of SMR in micro-polluted water body.

\subsection{The degradation mechanism of SMR removal by ozonation}

Three degradation intermediates were identified and denoted as $\mathrm{C} 1, \mathrm{C} 2$ and $\mathrm{C} 3$ according to the eluted sequence in the chromatograms (Table 1). C1, with a molecular ion of $m / z$ 156.1, could arise from the cleavage of the S-HN bond in SMR. C2, with a molecular ion of $m / z 109.1$, was identified to be 2-amino6-methylpyrimidine, which derived from the other half of the SHN bond break in SMR. C3, with a molecular ion of $m / z$ 93.1, was identified to be aniline, which derived from the cleavage of the $\mathrm{C}-\mathrm{S}$ bond in $\mathrm{C} 1 .^{45}$ No additional peaks appeared in the chromatogram after 8 minutes of reaction.

Overall, the degradation pathway of SMR during ozonation were proposed based on the three intermediates (Fig. 8). Firstly, S-HN bond cleavage to form $\left[\mathrm{C}_{6} \mathrm{H}_{6} \mathrm{NSO}_{2}\right]^{+}$and 2-amino-6methylpyrimidine $\left(\mathrm{C}_{5} \mathrm{H}_{7} \mathrm{~N}_{3}\right)$. Then, the $\mathrm{S}-\mathrm{C}$ bond of $\mathrm{C} 1$ is

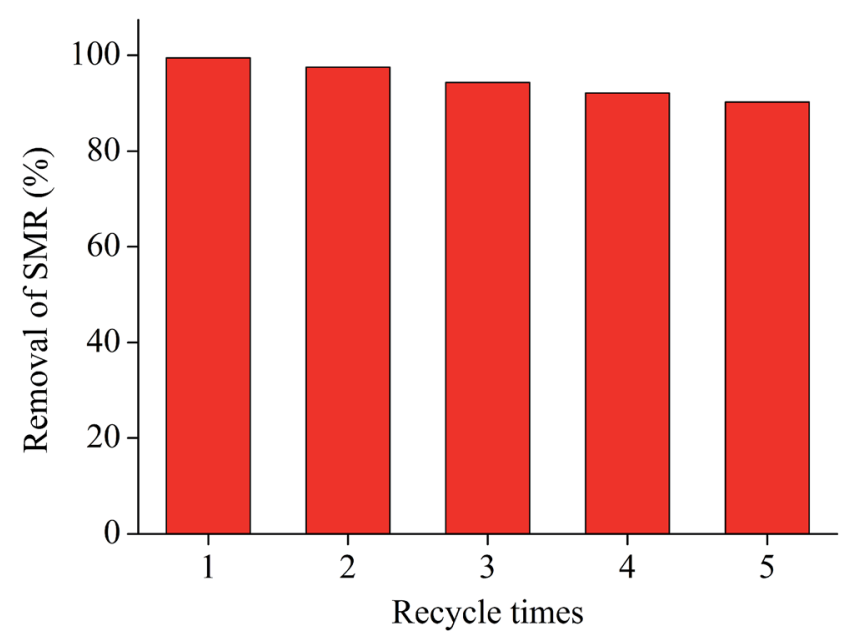

Fig. 7 Reusability of acetic acid solution for the removal of SMR. 
Table 1 Degradation product of SMR by ozonation

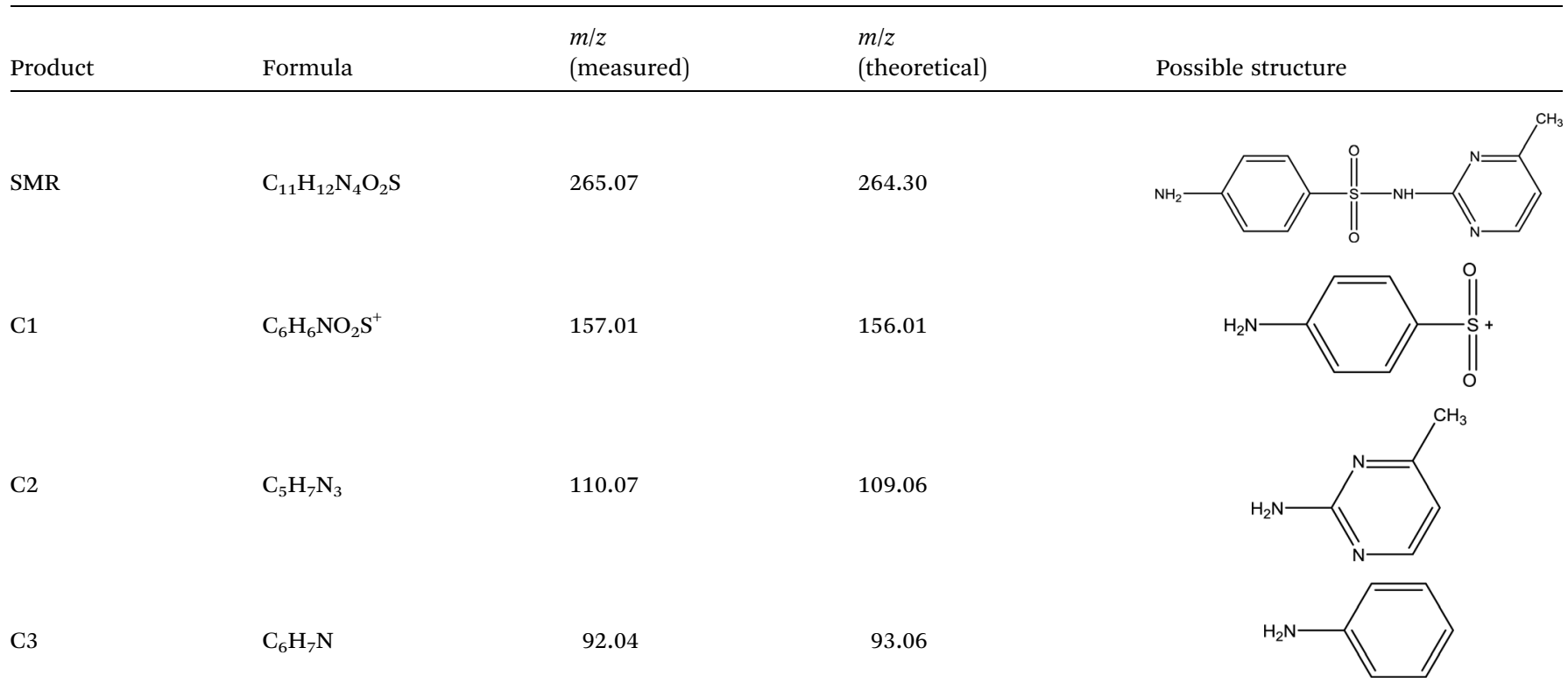

SMR, $\mathrm{m} / \mathrm{z}: 264.07$

Chemical Formula: $\mathrm{C}_{11} \mathrm{H}_{12} \mathrm{~N}_{4} \mathrm{O}_{2} \mathrm{~S}$

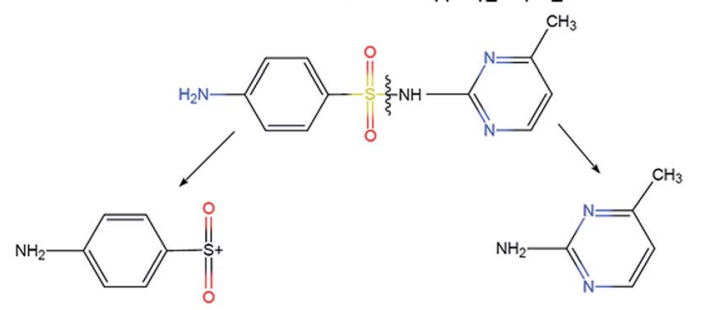

$\mathrm{Cl}, \mathrm{m} / \mathrm{z}: 156.01(100.0 \%)$

Chemical Formula: $\mathrm{C}_{6} \mathrm{H}_{6} \mathrm{NO}_{2} \mathrm{~S}^{+}$

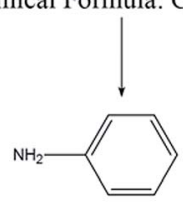

C2, $\mathrm{m} / \mathrm{z}: 109.6(100.0 \%$ Chemical Formula: $\mathrm{C}_{5} \mathrm{H}_{7} \mathrm{~N}_{3}$

$\mathrm{C} 3, \mathrm{~m} / \mathrm{z}: 93.06$

Chemical Formula: $\mathrm{C}_{6} \mathrm{H}_{7} \mathrm{~N}$

Fig. 8 Proposed degradation pathways of SMR during ozonation.

cleaved to form aniline $\left(\mathrm{C}_{6} \mathrm{H}_{7} \mathrm{~N}\right)$ and sulfate ions. Finally, $\mathrm{C} 2$ and $\mathrm{C} 3$ are further decomposed in a strong oxidation system.

\section{Conclusion}

The following conclusions can be drawn: GAC can be used as an adsorbent to transfer SMR from water to acetic acid. Acetic acid solution is a better desorbing agent for GAC carried with SMR, and a better reaction medium for SMR removal by ozonation. Acetic acid solution and GAC can be recycled. Ozonation of solutions of SMR in acetic acid allows the complete removal of this pollutant and also the depletion of the reaction intermediates. The removal rate of SMR in the AA solution decreases as the initial molar ratio of SMR and $\mathrm{O}_{3}$ increases. The ozonation mechanism of SMR is dominated by both direct and indirect ozonation in acetic acid solution. $\mathrm{Fe}^{3+}$ can inhibit the degradation of SMR by ozone in an acetic acid solution. Combined GAC adsorption-desorption in AA solution and ozonation of the resulting solution is a very efficient process for the degradation of diluted wastes.

\section{Conflicts of interest}

There are no conflicts to declare.

\section{Acknowledgements}

We gratefully acknowledge financial support by the National Natural Science Foundation of China (Grant No. 41373111), Jiangsu Provincial Key Laboratory of Carbon and Nitrogen Cycle Processes and Pollution Control, and Jiangsu Center for Collaborative Innovation in Geographical Information Resource Development and Application.

\section{References}

1 E. Dimina, M. Akermanis and U. Dumpis, Proc. Latv. Acad. Sci., Sect. B, 2009, 63(4-5), 253.

2 V. Homem and L. Santos, J. Environ. Manage., 2011, 92(10), 2304.

3 V. Sharma, R. V. Kumar, K. Pakshirajan and G. Pugazhenthi, J. Powder Technol., 2017, 321, 259.

4 C. Reyes, J. Fernandez, J. Freer, M. A. Mondaca, C. Zaror, S. Malato and H. D. Man-silla, J. Photochem. Photobiol., A, 2006, 184(1-2), 141.

5 W. Baran, E. Adamek, A. Sobczak and A. Makowski, Appl. Catal., B, 2009, 90(3-4), 516. 
6 J. D. Orwa, J. W. Matofari, P. S. Muliro and P. Lamuka, International Journal of Food Contamination, 2017, 4(1), 5.

7 R. Li, Y. L. Zhang, W. L. Chu, Z. X. Chen and J. L. Wang, RSC Adv., 2018, 8(24), 13546-13555.

8 H. M. Khan, H. Bae and J. Y. Jung, J. Hazard. Mater., 2010, 181(1-3), 659.

9 M. R. Azhar, H. R. Abid, H. Periasamy, M. O. Sun, V. Tadé and S. B. Wang, J. Colloid Interface Sci., 2017, 500(15), 88.

10 M. C. Ncibi and M. Sillanpää, J. Hazard. Mater., 2015, 298, 102.

11 I. Michael, E. Hapeshi, C. Michael, A. R. Varela, S. Kyriakou, C. M. Manaia, et al., Water Res., 2012, 46(17), 5621.

12 E. Elmolla and M. Chaudhuri, J. Hazard. Mater., 2009, 170(23), 666 .

13 E. Elmolla and M. Chaudhuri, J. Hazard. Mater., 2010, 173(13), 445 .

14 W. Li, X. Peng, H. Wang, Z. Liang and Z. Luan, Fresenius Environ. Bull., 2011, 20(1), 93-98.

15 M. Mehrjouei, S. Müller and D. Möller, Chem. Eng. J., 2015, 263(1), 209.

16 G. Moussavi, A. Alahabadi, K. Yaghmaeian and M. Eskandari, Chem. Eng. J., 2013, 217(2), 119.

17 J. Rivera-Utrilla, G. Prados-Joya, M. Sánchez-Polo, M. A. Ferro-García and I. Bautista-Toledo, J. Hazard. Mater., 2009, 170(1), 298.

18 J. Chen, J. P. Wang and G. C. Zhang, Chin. J. Environ. Eng., 2012, 6(12), 4493.

19 Z. Aksu and O. Tunç, Process Biochem., 2005, 40(2), 831.

20 M. R. Azhar, H. R. Abid, H. Sun, V. Periasamy, M. O. Tadé and S. B. Wang, J. Colloid Interface Sci., 2016, 478, 344.

21 M. B. Ahmed, J. L. Zhou, H. H. Ngo and W. Guo, Sci. Total Environ., 2015, 532, 112.

22 S. Xie, J. Y. Li and Q. X. Zhao, Chin. J. Environ. Eng., 2012, 6(2), 483.

23 A. R. Rahmani, D. Nematollahi, M. R. Samarghandi, M. T. Samadi and G. Azarian, J. Electroanal. Chem., 2018, 808, 82.

24 D. B. Mawhinney, B. J. Vanderford and S. A. Snyder, Environ. Sci. Technol., 2012, 46(13), 7102.
25 C. V. Gómez-Pacheco, M. Sánchez-Polo, J. Rivera-Utrilla and J. López-Penalver, Chem. Eng. J., 2011, 178, 115.

26 D. B. Ward, C. Tizaoui and M. J. Slater, Ozone: Sci. Eng., 2004, 26(5), 475-486.

27 J. Nawrocki and B. Kasprzyk-Hordern, Appl. Catal., B., 2010, 99(1), 27.

28 G. Li, W. W. Ben, H. Ye, D. Zhang and Z. M. Qiang, Chem. Eng. J., 2018, 341, 327-334.

29 D. B. Ward, C. Tizaoui and M. J. Slater, J. Hazard. Mater., 2005, 125(1-3), 65-79.

30 Y. Nakano, K. Okawa, W. Nishijima and M. Okada, Water Res., 2003, 37(11), 2595.

31 K. Okawa, M. Sasaki, Y. Nakano, W. Nishijima and M. Okada, J. Jpn. Soc. Water Environ., 2003, 26(12), 855.

32 S. Y. Li, T. Mei, Y. Peng, S. Y. Ge, G. X. Wang, Y. P. Zhu, L. X. Ni and Y. Zhang, Ozone: Sci. Eng., 2015, 37(5), 405.

33 Y. Liu, X. H. Liu, W. P. Dong, L. L. Zhang, Q. Kong and W. L. Wang, Sci. Rep., 2017, 7, 12437.

34 M. Naushad, T. Ahamad, B. M. Al-Maswari, A. A. Alqadami and S. M. Alshehri, Chem. Eng. J., 2017, 330, 1351-1360.

35 I. Arslan-Alaton and S. J. Dogruel, J. Hazard. Mater., 2004, 112(1-2), 105.

36 A. Goi, Y. Veressinina and M. Trapido, Ozone: Sci. Eng., 2009, 31(1), 28.

37 N. M. Mahmoodi and M. Arami, J. Photochem. Photobiol., A, 2006, 182(1), 60-66.

38 C. J. Wang, N. Klamerth, S. A. Messele, A. Singh, M. Belosevic and M. G. El-Din, Water Res., 2016, 100, 476-485.

39 U. von-Gunten, Water Res., 2003, 37(7), 1443.

$40 \mathrm{~J}$. Staehelin and J. Hoigne, Environ. Sci. Technol., 1985, 19(12), 1206.

41 K. Okawa, T. Y. Tsai, Y. Nakano, W. Nishijima and M. Okada, Chemosphere, 2005, 58(4), 523.

42 Y. Guo, H. J. Wang, B. Wang, S. B. Deng, J. Huang, Y. Gang and Y. J. Wang, Water Res., 2018, 142, 383.

43 S. Cortés, J. Sarasa, P. Ormad, R. Gracia and J. L. Ovelleiro, Ozone: Sci. Eng., 2000, 22(4), 415-426.

44 A. Albert and C. W. Rees, Nature, 1956, 177(4505), 433.

45 W. W. Ben, Y. W. Shi, W. W. Li, Y. Zhang and Z. M. Qiang, Chem. Eng. J., 2017, 327, 743. 